\title{
Percepção sobre a Diabetes Mellitus 2 na comunidade indígena Jabuti Bonfim - RR: um relato de experiência
}

\author{
Perception about Diabetes Mellitus 2 in the indigenous community Jabuti Bonfim - RR: an \\ experience report
}
Percepción sobre Diabetes Mellitus 2 en la comunidad indígena Jabuti Bonfim - RR: informe de experiencia

\begin{abstract}
Alessandra Tomaz Carioca ${ }^{1}$, Ana Paula Barbosa Alves ${ }^{1 *}$, Simone Lopes de Almeida ${ }^{1}$, Luiza Brum Argenta $^{1}$, Hosana Carolina dos Santos Barreto ${ }^{1}$, Marcos Antonio Braga de Freitas ${ }^{1}$.
\end{abstract}

\section{RESUMO}

Objetivo: Descrever a percepção das pessoas sobre a Diabetes Mellitus 2, as noções sobre saúde, doença e cuidados. Relato de experiência: Participaram da roda de conversa alguns usuários cadastrados no Programa HIPERDIA. Para os usuários, a saúde está relacionada a se viver bem na sua terra, poder trabalhar, sem nenhuma doença ou dor. E doença seria ter a diabetes, ter dores no corpo, não ter alegria para viver. Não sabiam explicar o que seria a diabetes. Todos sabiam identificar os sinais e sintomas de hipoglicemia e de hiperglicemia. Não praticavam atividades físicas regulares. Apontaram o atendimento dos profissionais de saúde, como seus principais cuidados de saúde. Fazem o uso de medicamentos prescritos pelos profissionais de saúde e de sua medicina tradicional. A principal dificuldade é manter uma dieta balanceada. Suas principais fortalezas são suas famílias e Deus. Quando pensam na diabetes, ficam tristes, tem medo de suas complicações e da morte. Considerações finais: Com essa atividade percebemos que a concepção de saúde-doença e cuidado no viver com a diabetes mellitus são diferentes para cada usuário, pois sofre influência de suas experiências pessoais, e guardam uma relação direta com as crenças, cultura e valores adquiridos ao longo das suas vidas.

Palavras chaves: Diabetes Mellitus tipo 2, Saúde de populações indígenas, Doença crônica.

\begin{abstract}
Objective: To describe people's perception of Diabetes Mellitus 2, the notions about health, disease, and care. Experience report: Some users registered in the Hiperdia Program participated in the conversation wheel. For users, health is related to living well in their land, to be able to work, without any illness or pain. And disease would be having diabetes, having body pain, not having joy to live. They did not know how to explain what diabetes would be. Everyone knew how to identify the signs and symptoms of hypoglycemia and hyperglycemia. They did not practice regular physical activities. They pointed to the care of health professionals, as their main health care. They use medicines prescribed by health professionals, and their traditional medicine. The main difficulty is maintaining a balanced diet. Their main strengths are their families and God. When they think about diabetes, they are sad, afraid of its complications and death. Final considerations: With this activity, we realized that the concept of health, disease and care in living with diabetes are different for each user, because it is influenced by their personal experiences, and they have a direct relationship with the beliefs, culture and values acquired throughout their lives.
\end{abstract}

Keywords: Diabetes Mellitus type 2, Health of indigenous peoples, Chronic disease.

1 Universidade Federal de Roraima, Boa Vista - RO. *E-mail: paula.alves@ufrr.br 


\section{RESUMEN}

Objetivo: Describir la percepción de las personas sobre la Diabetes Mellitus 2, las nociones sobre salud, enfermedad, y cuidados. Informe de experiencia: Algunos usuarios registrados en el Programa Hiperdia participaron en la rueda de conversación. Para los usuarios, la salud está relacionada con vivir bien en su tierra, poder trabajar sin ninguna enfermedad ni dolor. $\mathrm{Y}$ la enfermedad sería tener diabetes, tener dolor corporal, no tener alegría de vivir. No sabían cómo explicar qué sería la diabetes. Todos sabían cómo identificar signos y síntomas de hipoglucemia e hiperglucemia. No practicaban actividades físicas habituales. Señalaran la atención de los profesionales de la salud, como su principal atención sanitaria. Utilizan medicamentos recetados por profesionales de la salud y su medicina tradicional. La principal dificultad es mantener una dieta equilibrada. Sus principales fortalezas son sus familias y Dios. Cuando piensan en la diabetes, están tristes, temen sus complicaciones y la muerte. Consideraciones finales: Con esta actividad, nos dimos cuenta de que el concepto de salud, enfermedad y la forma en que cada usuario percibe la diabetes es diferente, ya que están influenciado por sus experiencias personales, y tiene una relación directa con las creencias, cultura y los valores adquiridos a lo largo de sus vidas.

Palabras clave: Diabetes Mellitus tipo 2, Salud de poblaciones indígenas, Enfermedad crónica.

\section{INTRODUÇÃO}

As doenças crônicas não transmissíveis (DCNT) são responsáveis por quase 40 milhões de óbitos em todo o mundo, expressando uma mortalidade prematura, abaixo de 70 anos de idade. No Brasil as DCNT simbolizam $75 \%$ dos óbitos sucedidos em todo país (MALTA DC, et al., 2017). Entre as DCNT se destaca a Diabetes Mellitus tipo 2 (DM 2) considerada como uma epidemia mundial, com alta taxas de morbimortalidade, em 20\% dos adultos entre 65 e 76 anos e efeitos socioeconômicos expressivos (VITOI NC, et al., 2015; SANTOS AL, et al., 2018).

A Sociedade Brasileira de Diabetes (2016) avalia que mundialmente existam 387 milhões de pessoas vivendo com diabetes e estima que em 2035 esse número aumente para 471 milhões. No Brasil, o número de pessoas com DM 2 em 2000, era de 4,5 milhões, e tornar-se-á 11,3 milhões em 2030 (SANTOS AL, et al., 2018). Segundo a International Diabetes Federation, o Brasil se encontra no 5o lugar no ranking mundial de pessoas que vivem com diabetes mellitus, sendo que em 2019 são 16, 8 milhões; e em 2030 serão 21,5 milhões, e 2045 serão 26,0 milhões de pessoas com DM (IDF GUÍA DE INCIDENCIA POLÍTICA, 2019).

Existem alguns fatores de risco para o surgimento da DM, como: história familiar da doença, etnia e aumento da idade, adoção de estilos de vida pouco saudáveis (sedentarismo, dieta inadequada, sobrepeso, obesidade), diagnóstico prévio de pré-diabetes ou diabetes mellitus gestacional, presença de hipertensão arterial e dislipidemia. A sua classificação é de acordo com a sua etiologia em: DM tipo 1, DM tipo 2, outros tipos específicos de DM e DM gestacional. Ainda, são consideradas duas categorias, mencionadas como prédiabetes: a glicemia de jejum alterada e a tolerância à glicose diminuída (SOCIEDADE BRASILEIRA DE DIABETES, 2016).

O viver com diabetes na maioria das vezes está relacionado ao aparecimento de complicações limitantes, que influenciam na prática das atividades do cotidiano, afetando a qualidade de vida das pessoas. Logo, quando a DM não é tratada ou controlada continuamente, os sinais e sintomas podem se agravar e, colaborar para o surgimento de outras patologias, como problemas cardíacos, visuais, acidente vascular cerebral, insuficiência renal, lesões de difícil cicatrização, dentre outras complicações (SANTOS IM, et al., 2020; SOCIEDADE BRASILEIRA DE DIABETES, 2016).

A incidência da DM nos povos indígenas no mundo é uma doença considerada emergente (FREITAS GA, et al., 2016). Está relacionada com problemas sociais que reverberam na posse do território e consequentemente na economia dessas sociedades, a introdução e o consumo de alimentos industrializados nas terras indígenas e na relação de contato cada vez mais intenso com o não-indígena. Doenças como diabetes, hipertensão, dislipidemias e obesidade, não eram prevalentes quando esses povos mantinham sua alimentação tradicional. Mas, hoje são uma realidade (ALVES APB, et al., 2019; BASTA PC, et al., 2012). 
Para Freitas GA, et al. (2016) afirmam que é preciso monitorar a evolução do DM entre os povos indígenas, procurando prevenir suas complicações e mortalidade prematuras. Assim, as ações de educação em saúde podem ser uma ferramenta importante para prevenção primária da DM e redução dos custos no setor saúde (FREITAS GA, et al., 2016; SALCI MA, et al., 2018).

Diante do aumento da prevalência da diabetes entre as sociedades indígenas ao longo dos anos, e da escassez de estudos locais, principalmente sobre os povos indígenas do Estado de Roraima, que permitam a comparação com os dados regionais e nacionais, este relato de experiencia é de suma importância, pois se trata de uma atividade em educação em saúde que buscou promover a qualidade de vida dessas populações. Assim, o objetivo deste relato foi descrever a percepção das pessoas sobre o DM 2, as noções sobre saúde, doença, cuidados.

\section{RELATO DE EXPERIÊNCIA}

Este relato foi fruto de uma roda de conversa realizada no Estágio. Essa experiência foi vivenciada pelas docentes e discente do Curso de Bacharelado em Gestão em Saúde Coletiva Indígena, do Instituto Insikiran de Formação Superior Indígena da Universidade Federal de Roraima (UFRR), no período de novembro e dezembro de 2018. A atividade foi possível devido o vínculo prévio com a comunidade a partir da discente que é moradora, pertencente a etnia Wapichana.

A comunidade Jabuti foi criada em meados dos anos 1970, com cinco famílias. Atualmente está com uma população estimada em 500 habitantes, sendo divididas em 105 famílias, pertencentes às etnias Macuxi e Wapichana. É administrada por um Tuxaua, que é a maior liderança dentro de uma comunidade indígena, contribui para ordenação, harmonização da vida cotidiana e representação de sua aldeia ou povo frente a outros povos (GERSEM SL, 2006). A sua demarcação ocorreu em 15 de fevereiro de 1996, sendo denominada de terra indígena Jabuti, com superfície de 14.210,6996 ha e perímetro de 63.018,61 m (BRASIL, 1996). O ambiente natural da região é característico de lavrados e pequenas matas ciliares (AMORIM, JEL, 2017).

Incialmente foi solicitada autorização para a realização da roda de conversa. Assim, as lideranças da comunidade em uma assembleia com os moradores aprovavam a execução da atividade. Neste sentido, a roda de conversa foi delineada para acontecer em um único momento, se houvesse necessidade, seria realizada outra roda com o consentimento prévio dos moradores. Basicamente, foi feito um planejamento, em que foram realizadas visitas nas casas dos moradores, para convidá-los a participar da ação, explicar o seu objetivo, e acordar o melhor dia e horário para ser realizada.

Depois, de escolher o dia e o horário, esboçamos a metodologia da roda: primeiramente fizemos uma dinâmica de apresentação, depois explicamos o objetivo da atividade, em seguida descrevemos as questõeschaves para disparar os diálogos, que foram: O que se entende sobre saúde e doença? O que sabemos sobre DM, seus cuidados e a prevenção das suas complicações? Quais seriam as principais dificuldades, fraquezas e fortalezas (apoio) para conviver com a doença?

Participaram da roda de conversa usuários cadastrados no Programa HIPERDIA. Participaram homens e mulheres, com idades entre 49 a 67 anos, que convivem com a diabetes entre 2 a 9 anos. A roda de conversa aconteceu no último sábado do mês de novembro de 2018.

As concepções sobre saúde e doença referidas pelo grupo foram que ter saúde é viver bem na sua terra, poder trabalhar, plantar e cuidar dos animais, sem nenhuma doença ou dor, poder realizar suas atividades cotidianas, sem ficar cansado. Logo, expressaram que doença seria ter a diabetes, ter dores na coluna, nas pernas, e na cabeça, é ter uma visão ruim, sem entusiasmo, seria não ter alegria para viver.

Sobre os significados e sentidos de viver com a DM Tipo 2, algumas pessoas não sabiam explicar basicamente o que é DM 2, expressaram que não tinham conhecimento. Ou demonstravam uma confusão entre a DM tipo 1 e a DM tipo 2, relataram que o médico da Equipe Multidisciplinar de Saúde Indígena (EMSI) tinha explicado que a pessoa já nascia com a diabetes, e que com o passar do tempo, ela apresentava 
alterações, dependendo dos hábitos de vida, como não fazer exercícios físicos, comer muito doce. Percebem que essa doença nunca existiu em sua família, sendo eles os primeiros casos entre seus parentes.

Todos sabem identificar os sinais e sintomas quando a glicose está baixa (hipoglicemia) ou quando está alta (hiperglicemia), relataram que quando apresentam hiperglicemia, sentem tonturas, dores de cabeça, fraqueza, escurece a vista, urinam muito e quando estão com hipoglicemia, sentem sono, fraqueza, suor frio, tremores, palpitações no peito, confusão mental, ansiedade.

A principal dificuldade destacada pelos usuários no controle da HAS e DM é manter uma dieta balanceada sem o uso do sal e açúcar. Identificaram que o cuidado com a alimentação é importante para a saúde.

Afirmaram que quando comiam sua comida tradicional não tinha essa doença entre eles. A maioria associa a DM 2 com a alimentação, pois comiam carne gorda, doces, frituras, calabresa, carne de porco, refrigerantes e outros. E alguns passaram a comer peixe, frango, carne (cozida ou assada), farinha, arroz, café com leite (com pouco açúcar), verduras (quando tem), batata doce, abobora, beiju e outros.

A maioria referiu não praticar atividades físicas regulares. Acreditam que cuidar da casa, capinar, limpar o quintal, molhar plantas, cuidar do gado, andar de bicicleta, já seria um tipo atividade física. Afirmam que as suas rotinas diárias mudaram, e a principal mudança foi referente ao trabalho, muitos não trabalham mais em suas roças e não fazem mais farinha, devido precisar ficar exposto ao sol quente e a fumaça, e sentirem falta de ar.

Os usuários apontaram o atendimento da EMSI, como seus principais cuidados de saúde. Em que fazem o controle da DM e recebem as medicações. Afirmam que precisam da ajuda dos profissionais de saúde, e procuram seguir as orientações no seu autocuidado.

Todos asseguraram que tomam os seus remédios no horário certo, e que as medicações os ajudam a conviver com a doença, e quando a medicação acaba e passam mais de dois dias sem tomá-las sentem fraqueza e tonturas. Além dos medicamentos farmacológicos, todos tomam remédios tradicionais.

Expressaram que quando pensam na diabetes, surge um sentimento de tristeza, o medo da morte, de ficar cego, de perder uma perna, pois sabem que não tem cura e que terão que conviver com ela na vida inteira. E em relação a principal fortaleza para ajudá-los a conviver com a DM, a maioria respondeu que a família era seu maior apoio. Em relação às crenças e conhecimentos, alguns acreditam que "através da fé em Deus, seriam curados por que não nasceram com a doença".

\section{DISCUSSÃO}

O entendimento sobre saúde e doença, e a forma que cada sujeito enfrenta seus padecimentos são desdobramentos de suas vivências particulares, relacionadas com suas construções socioculturais e valores obtidos ao longo da vida. Assim, as compreensões de doença são diferentes para cada pessoa (SANTOS AL, et al., 2018). Souza MLP (2012), afirma que para efetiva promoção da saúde, a assistência em saúde necessita admitir e adotar as percepções populares sobre o processo saúde-doença-cuidado.

Os termos "doença" e "enfermidade" são conceitos distintos, usados por profissionais para descrever as diferentes perspectivas da saúde debilitada das pessoas. As doenças são as chamadas entidades patológicas que compõem o modelo médico de saúde debilitada, como a diabetes, e que podem ser especificamente identificadas e descritas pela referência a certa evidência biológica, química ou outra. Já, a enfermidade é a perspectiva do paciente sobre sua saúde debilitada, o que é muito diferente do modelo de doença (HELMAN CG, 2009).

Resende JM (2014), em seu estudo enfatiza os termos afecção, doença, enfermidade, moléstia que apresentam uma diferença semântica e consisti em um importante objeto de investigação na área linguística como no campo da medicina, e são na atualidade usados como sinônimos de forma equivocada. Destarte, que etimologicamente, o doente é o que experimenta a dor, a aflição, o padecimento; o enfermo é o que está debilitado, enfraquecido pela doença. 
Corrêa K, et al. (2017), manifestam que algumas condições podem interferir na qualidade de vida das pessoas que convivem com a DM, como por exemplo: o tipo de DM, uso de insulina, idade, complicações, nível social, fatores psicológicos, etnias, educação, conhecimento sobre a doença, tipo de assistência, entre outras. É importante que o usuário, a família e a equipe de saúde, saibam identificar sinais de alterações, complicações do DM, e quando e onde devem buscar cuidados de saúde emergências para evitar complicações e a morte prematura (BRASIL MS, 2006).

O convívio com a DM solicita um novo viver em busca de bem-estar (BRASIL MS, 2006). Se faz necessário adequar novas práticas ao estilo de vida, como por exemplo, a ingesta de medicamentos, alterações alimentares, realização de controle glicêmico, prática frequente de atividades físicas, manutenção dos níveis pressóricos, e o acompanhamento contínuo da equipe multiprofissional de saúde (SANTOS AL, et al., 2018).

Duncan BB, et al. (2012) asseguram que a DCNT, ocasionam uma diminuição da qualidade de vida, causando uma redução das ações laborais e de lazer e, podem ampliar a mortalidade prematura. Além disso, impõem custos econômicos as famílias, comunidades e a sociedade em geral. A atividade física é reconhecida como fator de proteção para a saúde, sendo seus benefícios associados à redução de doenças crônicas e à diminuição do risco de morte prematura por doenças cardiovasculares (POLISSENI MLC e RIBEIRO CR, 2014).

Em relação ao uso dos medicamentos, se percebeu a integração por partes das pessoas do conhecimento biomédico e da medicina tradicional. Nos estudos de Scopel D, et al. (2015), a intermedicalidade são práticas que articulam vários saberes, como os populares, os tradicionais e os biomédicos para manter a boa saúde e tratar às enfermidades.

O cumprimento da prescrição médica é um fenômeno complexo que se constitui mais do que cumprir algumas imposições. No procedimento de adesão, é preciso negociar com o paciente, se faz necessário favorecer a sua autonomia para conseguir ajustar às prescrições indicadas pelos profissionais de saúde, sendo o usuário o protagonista de seu autocuidado (PONTIERI FM e BACHIN MM, 2010).

O sentimento de tristeza nas pessoas que vivem com a DM pode prejudicar o manejo da doença. Assim, o profissional de saúde precisa buscar estratégias de apoio psicossocial e aplicar um grande esforço para sensibilizar sobre os benefícios da continuação adequada do tratamento prescrito, o qual poderá proporcionar uma melhor qualidade de vida (COSTA JA, et al., 2011).

Para o convívio com a doença o apoio familiar e a crença em Deus são fundamentais. A família atua como uma rede de apoio para o cuidado e acolhimento, colabora para que a pessoa receba melhor a sua nova condição e, tenha comportamentos otimistas e eficientes no autocuidado (SANTOS AL e MARCON SS, 2014). A fé em Deus, ajuda na continuidade do tratamento. Contribuindo para o fortalecimento de sua autoestima, relações sociais e familiares, na redução do risco de complicações e do óbito prematuro (LUENGO CML e MENDONÇA ARA, 2014).

A DM é uma doença de complexo enfrentamento. Assim as estratégias de educação em saúde são de suma importância para seu manejo e controle, para que as pessoas consigam ou preservem sua qualidade de vida (CORRÊA K, et al., 2017). Vale apontar que o uso de metodologias ativas como a roda de conversa podem ser um potente instrumento para a promoção da saúde (DIAS ESM, et al., 2018).

As rodas de conversa por Paulo Freire chamadas "Círculos de Cultura", favorecem ocasiões de fala e de escuta. Em que podemos apreender o mundo, problematizá-lo, compreendê-lo e transformá-lo. Consistir em um bate-papo, em que a reflexão do instrutor apenas recebe legitimidade, na verdade da razão dos aprendizes, ambos em um processo de comunicação influenciados pela realidade, logo na comunicação recíproca de um para outro (FREIRE $P, 2003$ ).

Com essa atividade percebemos que a concepção de saúde-doença e cuidado no viver com a DM são diferentes para cada usuário, pois sofre influência de suas experiências pessoais, e guardam uma relação direta com as crenças, cultura e valores adquiridos ao longo das suas vidas. As pessoas tentam estabelecer seu autocuidado a partir do viver com a diabetes, buscando um melhorar a alimentação, dando continuidade ao tratamento biomédico e de sua medicina tradicional. 


\section{REFERÊNCIAS}

1. ALVES APB, et al. Doenças e agravos mais prevalentes em uma comunidade indígena em Boa Vista-RR: relato de experiência. Revista Eletrônica Acervo Saúde, 2019; (26); e673.

2. AMORIM JEL. Geoambiental characteristics of the Igarapé água boa do bom intento, Boa Vista, Roraima. Geosaberes, 2017; 9(17): 1 -13.

3. BASTA PC, et al. Perfil epidemiológico dos povos indígenas no Brasil: notas sobre agravos selecionados. In: Garnelo L, Pontes, AL (Org.). Saúde Indígena: uma introdução ao tema. Brasília: MEC-SECADI, 2012; 60-108p.

4. BRASIL. Ministério da Saúde. Secretaria de Atenção à Saúde. Departamento de Atenção Básica. Diabetes Mellitus. Brasília: Ministério da Saúde, 2006; 64p. Disponível em: https://bvsms.saude.gov.br/bvs/publicacoes/diabetes_mellitus.PDF. Acesso em: 03/02/2020.

5. BRASIL. Ministério da Saúde. Secretaria de Atenção à Saúde. Departamento de Atenção Básica. Envelhecimento e saúde da pessoa idosa. Brasília: Ministério da Saúde, 2006; 192p. Disponível em: https://bvsms.saude.gov.br/bvs/publicacoes/evelhecimento_saude_pessoa_idosa.pdf. Acesso em: 03/02/2020.

6. BRASIL. Decreto de 15 Fevereiro de $1996 . \quad$ Disponível https://acervo.socioambiental.org/sites/default/files/documents/0BD00225.pdf. Acesso em: 03/02/2020.

7. COSTA JA, et al. Promoção da saúde e diabetes: discutindo a adesão e a motivação de indivíduos diabéticos participantes de programas de saúde. Ciênc. saúde coletiva, 2011; 16(3): 2001-2009.

8. CÔRREA K, et al. Qualidade de vida e características dos pacientes diabéticos. Ciênc. saúde coletiva, 2017; 22(3): 921-930.

9. DIAS ESM, et al. Roda de conversa como estratégia de educação em saúde para a enfermagem. Revista de Pesquisa: Cuidado é fundamental online, 2018; 10(2): 379-384.

10. DUNCAN BB, et al. Doenças Crônicas Não Transmissíveis no Brasil: prioridade para enfrentamento e investigação. Revista de Saúde Pública, 2012; 46(Supl): 126-34.

11. FREITAS GA, et al. Prevalência de diabetes mellitus e fatores associados em mulheres indígenas do Município de Dourados, Mato Grosso do Sul, Brasil. Cadernos de Saúde Pública, 2016; 32(8): e00023915.

12. FREIRE, P. Pedagogia do Oprimido. $36^{a}$ ed. Rio de Janeiro: Paz e Terra, $2003 ; 184 p$.

13. GARNELO L. Política de Saúde Indígena no Brasil: notas sobre as tendências atuais do processo de implantação do subsistema de atenção à saúde. In: Garnelo L, Pontes, AL (Org.). Saúde Indígena: uma introdução ao tema. Brasília: MEC-SECADI, 2012; 18-59 p.

14. GERSEM SL. O Índio Brasileiro: o que você precisa saber sobre os povos indígenas no Brasil de hoje. Brasília: Ministério da Educação, Secretaria de Educação Continuada, Alfabetização e Diversidade. LACED/Museu Nacional, 2006; 224 p.

15. HELMAM CG. Doença versus enfermidade na clínica geral. Universidade de College, Londres. Tradução. Campos, 2009, 10(1):119-128.

16. IDF. INTERNATIONAL DIABETES FEDERATION. GUÍA DE INCIDENCIA POLÍTICA DE LA NOVENA EDICIÓN DEL ATLAS DE LA DIABETES DE LA FID 2019. Disponível em: https://www.diabetesatlas.org/upload/resources/material/20200121_115939_2407-IDF-Advocacy-Guide-SP-Finallowres-210120.pdf. Acesso em 29/12/2020.

17. LUENGO CML, MENDONCA ARA. Espiritualidade e qualidade de vida em pacientes com diabetes. Revista Bioética, 2014; 22(2): 380-387.

18. MALTA DC, et al. Doenças crônicas não transmissíveis e a utilização de serviços de saúde: análise da Pesquisa Nacional de Saúde no Brasil. Revista de Saúde Pública, 2017; 51(Supl 1): 4s.

19. MARASCHIN JF, et al. Classificação do diabete melito. Arquivos Brasileiros de Cardiologia, 2010; 95 (2): $40-46$.

20. MCLELLAN KCP, et al. Diabetes mellitus do tipo 2, síndrome metabólica e modificação no estilo de vida. Revista de Nutrição, 2007; 20(5): 515-524.

21. ONU. United Nations Transforming Our World: the 2030 Agenda for Sustainable Development. A/RES/70/1. United Nations. 2015.

22. POLISSENI MLC, RIBEIRO LC. Exercício físico como fator de proteção para a saúde em servidores públicos. Revista Brasileira de Medicina do Esporte, 2014; 20(5): 340-344.

23. PONTIERI FM, BACHIN MM. Crenças de pacientes diabéticos acerca da terapia nutricional e sua influência na adesão ao tratamento. Ciênc. saúde coletiva, 2010; 15(1): 151-160.

24. REZENDE MJ. AFECČÃO, DOENÇA, ENFERMIDADE, MOLÉSTIA. Revista de Patologia Tropical/Journal of Tropical Pathology, 2014; $43(3): 384-388$.

25. SANTOS AL, MARCON SS. Como as pessoas com diabetes avaliam a participação familiar em seu processo de cuidado da saúde. Investigación y Educación en Enfermería, 2014; 32(2): 260-269.

26. SANTOS AL, et al. Viver e conviver com diabetes: dificuldades experienciadas no enfrentamento e manejo da doença. Revista de Enfermagem UERJ, 2018; 26: e18221.

27. SANTOS IM, et al. Conhecimento e atitudes de usuários com Diabetes Mellitus em uma unidade de ambulatório especializada. Revista Eletrônica Acervo Saúde, 2020; 12(12): e4148.

28. SCOPEL D, et al. Intermedicalidade e protagonismo: a atuação dos agentes indígenas de saúde Munduruku da Terra Indígena Kwatá-Laranjal, Amazonas, Brasil. Cadernos de Saúde Pública, 2015; 31(12):2559-2568.

29. SILVA ECA, Povos indígenas e o direito à terra na realidade brasileira. Serviço Social \& Sociedade, 2018; (133): 480500 .

30. SOCIEDADE BRASILEIRA DE DIABETES (SBD). Diretrizes da Sociedade Brasileira de Diabetes (2015-2016). São Paulo: AC Farmacêutica, 2016.

31. SOUZA MLP. Da prevenção de Doenças à promoção de saúde: reflexões a partir da questão do uso de bebidas alcoólicas por populações indígenas. İn: Garnelo L, Pontes, AL (Org.). Saúde Indígena: uma introdução ao tema. Brasília: MEC-SECADI, 2012; 108-127p.

32. VITOI NC, et al. Prevalência e fatores associados ao diabetes em idosos no município de Viçosa, Minas Gerais. Revista Brasileira de Epidemiologia, 2015; 18(4): 953-965. 